\title{
$\mathbb{A}$ Economics Bulletin
}

\section{Volume 30, Issue 1}

\section{Implied volatility and risk aversion in a simple model with uncertain growth}

Frederik Lundtofte

Dept. of Economics, Lund University

\begin{abstract}
We show that a simple equilibrium model with uncertain growth is able to simultaneously generate patterns in implied volatility and risk aversion that are similar to the ones observed in the data.
\end{abstract}

I thank Nicole Branger, David Feldman, Fabio Trojani, Erik Lindström, and the participants at the 2005 Arne Ryde Workshop (Lund) and Risk Management: From Basel II to Basel III (Ascona) for helpful discussions. Financial support from the Jan Wallander and Tom Hedelius Foundation (grant W2005-0365:1) and the Swiss National Science Foundation (NCCR FINRISK and grant 100012-105745/1) is gratefully acknowledged.

Citation: Frederik Lundtofte, (2010) "Implied volatility and risk aversion in a simple model with uncertain growth ", Economics Bulletin, Vol. 30 no.1 pp. 182-191.

Submitted: Feb 27 2009. Published: January 13, 2010. 


\section{Introduction}

We use a simple equilibrium model of parameter uncertainty to simultaneously generate patterns in implied volatility and risk aversion that are similar to those observed empirically, i.e., skews in implied volatilities, smiles in implied risk aversions, and negative values on implied risk aversions (Corrado and Su, 1997; Jackwerth, 2000).

The stock return distribution becomes a mixture of lognormals, and the paper is thus related to Brigo, Mercurio and Rapisarda (2004). In Brigo, Mercurio and Rapisarda (2004), the stock return process is exogenously assumed. However, we present an equilibrium model in which the stock return process is endogenously determined. In contrast to Brigo, Mercurio and Rapisarda (2004), the stock return volatility is a known constant in this model, and uncertainty concerns the growth rate in the economy -- an inherently fundamental economic quantity.

Our modeling induces a special form of state dependence on the stochastic discount factor. Thus, it is related to the works of Brown and Jackwerth (2000), Garcia, Luger and Renault (2001), Garcia, Ghysels and Renault (2007), and Chabi-Yo, Garcia and Renault (2008). The contribution of this paper is to construct a simple model with a state-dependent stochastic discount factor that is able to generate patterns in both implied volatility and risk aversion that are in line with empirical observations.

\section{The Model}

We consider a Lucas (1978) exchange economy with an infinitely-lived representative consumer, who has constant relative risk aversion $\gamma>0$. His instantantaneous utility of consumption is given by $u(c)=\left(c^{1-\gamma}-1\right) /(1-\gamma)$, and he maximizes his expected life-time utility of consumption, $E_{t}\left[\int_{s=t}^{\infty} e^{-\beta s}\left(\frac{c_{s}^{1-\gamma}-1}{1-\gamma}\right) d s\right]$.

The aggregate endowments $\left(D_{t}\right)$ evolve according to the following SDE:

$$
\frac{d D_{t}}{D_{t}}=\left\{\begin{array}{c}
\theta_{0} d t+\sigma_{D} d W_{t} t \in[0, \varepsilon) \\
\theta d t+\sigma_{D} d W_{t} \quad t \geq \varepsilon
\end{array}\right.
$$

where $\varepsilon>0$ is an infinitesimal constant, $\theta_{0}$ is a constant, $\sigma_{D}$ is a positive constant, and $W_{t}$ is a standard Brownian motion. Further, for $t \in[0, \varepsilon), \theta$ is a stochastic variable, independent of $W_{t}$, with the following distribution:

$$
\theta=\left\{\begin{array}{c}
\theta_{1} \text { with probability } \mathrm{v}_{1} \\
\theta_{2} \text { with probability } \mathrm{v}_{2} \\
" \\
\theta_{n} \text { with probability } \mathrm{v}_{n}
\end{array}\right.
$$

where $v_{i}>0(\mathrm{i}=1,2, \ldots, \mathrm{n})$, and probabilities sum to one, $\sum_{i=1}^{n} v_{i}=1$. At time $t=\varepsilon$, the representative consumer knows the value of the growth rate $\theta$.

An interpretation of this set-up is that the representative consumer works with a number of scenarios for the growth rate, which is revealed to him at time $t=\varepsilon$. Thus, we can interpret our model as a stylized model of growth uncertainty. 


\section{Theoretical Results}

In this section, we solve for the equilibrium and present some useful results regarding the implied risk aversion.

\subsection{Equilibrium}

Since this is a pure exchange economy with a perishable consumption good, aggregate consumption must equal aggregate dividends, and a pricing kernel is thus given by

$$
\Lambda_{t}=e^{-\beta t} D_{t}^{-\gamma}
$$

(Cochrane, 2001, pp. 28-33).

Using Ito's Lemma on equation (1), we can determine the risk free rate of return in the economy.

Proposition 1: The interest rate, $r_{t}$, is given by

$$
r_{t}=\left\{\begin{array}{c}
\beta+\gamma \theta_{0}-(1+\gamma) \frac{\gamma \sigma_{D}^{2}}{2} t \in[0, \varepsilon) \\
\beta+\gamma \theta-(1+\gamma) \frac{\gamma \sigma_{D}^{2}}{2} t \geq \varepsilon
\end{array}\right.
$$

Proof. The result follows from the fact that the risk free rate is given by $r_{t}=-\frac{1}{d t} E_{t}\left[\frac{d \Lambda_{t}}{\Lambda_{t}}\right]$ (Cochrane, 2001, pp. 28-33) and an application of Ito's Lemma.

Given that the representative agent's time discount factor $(\beta)$ is high enough, we can price the claim to the entire stream of aggregate endowments, which can be interpreted as a stock.

Proposition 2: Given that $\beta>(1-\gamma)\left(\theta_{i}-\frac{\gamma}{2} \sigma_{D}^{2}\right)$ for all $i$, the price of the claim to the entire stream of endowments (the stock) is

$$
S(t)=\left\{\begin{array}{c}
D_{t} \sum_{i=1}^{n} v_{i} Q_{i} t \in[0, \varepsilon) \\
D_{t} Q t \geq \varepsilon
\end{array},\right.
$$

where $Q_{i}=\frac{1}{\beta-(1-\gamma)\left(\theta_{i}-\frac{\gamma}{2} \sigma_{D}^{2}\right)}$ and $Q=\frac{1}{\beta-(1-\gamma)\left(\theta-\frac{\gamma}{2} \sigma_{D}^{2}\right)}$.

Proof. Consider the price of the endowment stream from time $t$ to time $\tau$ at time $t \in[0, \varepsilon)$ (where $\tau>\varepsilon$ ). Applying the law of iterated expectations and Fubini-Tonelli's theorem, we have that

$$
\begin{aligned}
& S(t)=E_{t}\left[\int_{s=t}^{\tau} \frac{\Lambda_{s}}{\Lambda_{t}} D_{s} d s\right]=E_{t}\left[\int_{s=t}^{\varepsilon} \frac{\Lambda_{s}}{\Lambda_{t}} D_{s} d s\right]+E_{t}\left[\int_{s=\varepsilon}^{\tau} \frac{\Lambda_{s}}{\Lambda_{t}} D_{s} d s\right]=\int_{s=t}^{\varepsilon} E_{t}\left[\frac{\Lambda_{s}}{\Lambda_{t}} D_{s}\right] d s+ \\
& +E_{t}\left[E_{t}\left[\int_{s=\varepsilon}^{\tau} \frac{\Lambda_{s}}{\Lambda_{t}} D_{s} d s \mid \theta=\theta_{i}\right]\right]=D_{t} \int_{s=t}^{\varepsilon} e^{\left[-\beta+(1-\gamma)\left(\theta_{0}-\frac{\gamma}{2} \sigma_{D}^{2}\right)\right](s-t)} d s+E_{t}\left[\int_{s=\varepsilon}^{\tau} E_{t}\left[\frac{\Lambda_{s}}{\Lambda_{t}} D_{s} \mid \theta=\theta_{i}\right] d s\right]= \\
& =D_{t} \int_{s=t}^{\varepsilon} e^{\left[-\beta+(1-\gamma)\left(\theta_{0}-\frac{\gamma}{2} \sigma_{D}^{2}\right)\right](s-t)} d s+D_{t} \sum_{i=1}^{n} v_{i} \frac{1-e^{\left[-\beta+(1-\gamma)\left(\theta_{i}-\frac{\gamma}{2} \sigma_{D}^{2}\right)\right](\tau-\varepsilon)}}{\beta-(1-\gamma)\left(\theta_{i}-\frac{\gamma}{2} \sigma_{D}^{2}\right)} .
\end{aligned}
$$


As $\tau \rightarrow \infty$ and $\varepsilon \rightarrow 0, S(t) \rightarrow D_{t} \sum_{i=1}^{n} v_{i} Q_{i}$ (provided that $\beta-(1-\gamma)\left(\theta_{i}-\frac{\gamma}{2} \sigma_{D}^{2}\right)>0$ for all $i$ ).

If $t \geq \varepsilon$, the true growth rate is known to the representative consumer, and hence the price of the endowment stream is

$$
S(t)=E_{t}\left[\int_{s=t}^{\tau} \frac{\Lambda_{s}}{\Lambda_{t}} D_{s} d s\right]=\int_{s=t}^{\tau} E_{t}\left[\frac{\Lambda_{s}}{\Lambda_{t}} D_{s}\right] d s=D_{t} \frac{1-e^{\left[-\beta+(1-\gamma)\left(\theta-\frac{\gamma}{2} \sigma_{D}^{2}\right)\right](\tau-t)}}{\beta-(1-\gamma)\left(\theta-\frac{\gamma}{2} \sigma_{D}^{2}\right)}
$$

which goes to $D_{t} Q$ as $\tau \rightarrow \infty$ (provided that $\beta-(1-\gamma)\left(\theta_{i}-\frac{\gamma}{2} \sigma_{D}^{2}\right)>0$ for all $\left.i\right)$.

It is now easy to show that the instantaneous volatility of stock returns is constant and equals the volatility of dividends.

Corollary 3: The instantaneous volatility of the stock return, $\sigma_{S}$, is constant, and equals the volatility of dividends, $\sigma_{S}=\sigma_{D}$.

Proof. This result follows directly from the fact that the stock price is proportional to the current dividend (Proposition 2).

Due to uncertainty regarding the endowment growth rate, the time- 0 subjective density function for the stock price is a mixture of lognormals. As seen in equation (3), conditional stock prices differ not only in terms of growth rates, but also in terms of their time- $\varepsilon$ values (i.e. values immediately after time 0). Further, due to growth uncertainty, both the future interest rate and the future dividend yield are stochastic at time 0 , and depend on the value of the future endowment growth rate. As a result, the time-0 price of a European call option becomes a convex combination of Merton (1973) prices under the different growth rates, where the weights are given by the probabilities of the growth rates. Note that we consider a representative-agent equilibrium with a non-satiated representative agent. By the Fundamental Theorem of Asset Pricing, the thus obtained equilibrium prices are free of arbitrage (e.g., Dybvig and Ross, 2003, and the references therein).

Proposition 4: The time-0 equilibrium price of a European call option written on the stock, with strike price $K$ and time to maturity $T$, is given by

where

$$
C(S(0), T)=\sum_{i=1}^{n} v_{i} C_{i}(S(0), T),
$$

$$
\begin{aligned}
C_{i}(S(0), T)=\frac{S(0) Q_{i}}{\sum_{i=1}^{n} v_{i} Q_{i}} \exp \left\{-\left[\beta-(1-\gamma)\left(\theta_{i}-\frac{\gamma}{2} \sigma_{D}^{2}\right)\right] T\right\} \Phi\left(d_{i, 1}\right)+ \\
-K \exp \left\{-\left[\beta+\gamma \theta_{i}-(1+\gamma) \frac{\gamma \sigma_{D}^{2}}{2}\right] T\right\} \Phi\left(d_{i, 2}\right) \\
d_{i, 1}=\frac{1}{\sigma_{D} \sqrt{T}}\left(\ln (S(0) / K)+\ln \left(Q_{i} / \sum_{i=1}^{n} v_{i} Q_{i}\right)+\left(\theta_{i}-\frac{\sigma_{D}^{2}}{2}+(1-\gamma) \sigma_{D}^{2}\right) T\right) \\
d_{i, 2}=d_{i, 1}-\sigma_{D} \sqrt{T}
\end{aligned}
$$

Proof. Available from the author upon request. 
We define the implied volatility as the volatility which makes the Merton (1973) call price equal the equilibrium call price.

\subsection{Implied Risk Aversion}

Measures of implied risk aversion are based on the following relation, shown in Aït-Sahalia and Lo (2000):

$$
A_{r}\left(S_{T}\right)=\frac{p^{\prime}\left(S_{T}\right)}{p\left(S_{T}\right)} S_{T}-\frac{q^{\prime}\left(S_{T}\right)}{q\left(S_{T}\right)} S_{T},
$$

where $A_{r}\left(S_{T}\right)$ is the relative risk aversion, $p\left(S_{T}\right)$ is the subjective density function, $q\left(S_{T}\right)$ is the risk-neutral density function, and $S_{T}$ is the value of the stock. By definition, absolute risk aversion can be obtained by dividing relative risk aversion by $S_{T}$. Estimates of the riskneutral density function are usually based on the following finding in Breeden and Litzenberger (1978):

$$
q\left(S_{T}\right)=\left.e^{r(T-t)} \frac{\partial^{2} C}{\partial K^{2}}\right|_{K=S_{T}},
$$

where $C$ is the price of a call option maturing at time $T$, and $r$ is the interest rate.

Call prices are typically estimated for strike prices for which there exist no traded options. Practically, the estimated call prices are obtained from inter- and extrapolations of the observed implied volatility surface. Here, we are going to disregard the effects of these estimation errors, i.e., in the calibration, we are going to calculate theoretical option prices directly for a large number of strike prices, and use these option prices to determine the second derivative numerically as

$$
\frac{\partial^{2} C}{\partial K^{2}} \approx \frac{C(K+\Delta K, T)-2 C(K, T)+C(K-\Delta K, T)}{(\Delta K)^{2}} .
$$

\section{Calibration}

In this section, we calibrate the model to the data, and we study patterns of implied volatility and implied risk aversion. Guided by the empirical results regarding aggregate dividends summarized in Campbell, Lo and MacKinlay (1997), we set the volatility of aggregate dividends $\left(\sigma_{D}\right)$ to $12 \%$. In accordance with the results in Hagiwara and Herce (1997) and Basak and Cuoco (1998), we set the coefficient of relative risk aversion $(\gamma)$ to 3 . For simplicity, we assume that the growth rate can only attain two possible values, $\theta_{1}=2 \%$ or $\theta_{2}=3 \%$. That is, the expected growth rate is between 2 and $3 \%$, which roughly corresponds to the long-run real growth rate of the U.S. economy. We denote the probability of a low growth rate by $v$, and we set the time discount factor to $\beta=0.05$.

\subsection{Implied Volatility}

Given our definition of implied volatility as the volatility which makes the Merton (1973) call price equal the equilibrium call price, we follow most of the literature and solve for the implied volatility numerically. For an alternative way of solving for the implied volatility, we refer to Sukhomlin (2007).

Although the stock volatility is constant in our model, a wide range of implied volatility shapes can be generated, depending on the representative agent's beliefs (see Figures 1 to 3 ). 
The return distribution is a mixture of lognormals: a high (low) value on $v$ means that a relatively high (low) weight is put on the return distribution implied by the low growth rate.

For a high (low) value on $v$, the implied volatility exhibits a negatively (positively) sloped smirk pattern, whereas $v=0.5$ creates an implied volatility frown. One realistic property of the model is that the implied volatility shapes flatten out as time to maturity lengthens.

\subsection{Implied Risk Aversion}

In addition, we investigate the model implications for implied risk aversion. Since most empirical studies on implied volatility have found a negatively sloped smirk pattern, we focus on the case $v=0.9$, to see whether we simultaneously can explain the negative values and smile patterns that have been found in empirical studies on implied risk aversion, such as Jackwerth (2000).

Figure 4 shows that not only are we able to generate a smile pattern in implied risk aversion, but we are also able generate negative values. Figure 5 shows the corresponding implied pricing kernel as a function of the cash price $\left(S_{T}\right) .{ }^{1}$ We find that the implied pricing kernel is hump-shaped, i.e., for particular levels of wealth, it is in fact increasing. In the literature, this phenomenon is referred to as a "pricing kernel puzzle."

As Figure 6 illustrates, the measure developed by Aït-Sahalia and Lo (2000) of the implied risk aversion is very sensitive to misestimations of subjective beliefs.

\section{Conclusions}

By using a simple equilibrium model with uncertain growth, we are able to simultaneously generate patterns in implied volatility and risk aversion that are similar to what is observed empirically. The generated implied volatility shapes have the realistic property that they flatten out as time to maturity lengthens. We are also able to generate implied risk aversions that are in line with empirical studies, such as Jackwerth (2000). That is, we are able to produce both a smile pattern and negative values.

\footnotetext{
${ }^{1}$ Following Aït-Sahalia and Lo (2000), we calculate the implied pricing kernel at time 0 as $\varphi_{0, T}=e^{-r T} q\left(S_{T}\right) / p\left(S_{T}\right)$.
} 


\section{References:}

Aït-Sahalia, Y. and A. W. Lo (2000) "Nonparametric Risk Management and Implied Risk Aversion" Journal of Econometrics 94, 9-51.

Basak, S. and D. Cuoco (1998) "An Equilibrium Model with Restricted Stock Market Participation” Review of Financial Studies 11, 309-341.

Breeden, D. and R. Litzenberger (1978) "Prices of State-Contingent Claims Implicit in Option Prices" Journal of Business 51, 621-651.

Brown, D. P. and J. C. Jackwerth (2004) "The Pricing Kernel Puzzle: Reconciling Index Option Data and Economic Theory" Working paper, University of Konstanz.

Brigo, D., F. Mercurio and F. Rapisarda (2004) "Smile at the Uncertainty" Risk, May Issue, 97-101.

Campbell, J. Y., A. W. Lo and A. C. MacKinlay (1997) The Econometrics of Financial Markets, Princeton University Press: Princeton.

Chabi-Yo, F., R. Garcia and E. Renault (2008) "State Dependence Can Explain the Risk Aversion Puzzle" Review of Financial Studies 21, 973-1011.

Cochrane, J. H. (2001) Asset Pricing, Princeton University Press: Princeton.

Corrado, C. J., and T. Su (1997) "Implied Volatility Skews and Stock Index Skewness and Kurtosis Implied by S\&P 500 Index Option Prices" Journal of Derivatives 4, 8-19.

Dybvig, P. H. and S. A. Ross (2003) "Arbitrage, State Prices and Portfolio Theory" in Handbook of the Economics of Finance by G. M. Constantinides, M. Harris and R. M. Stulz, Eds., North-Holland: Amsterdam, 605-637.

Garcia, R., E. Ghysels and E. Renault (2007) "The Econometrics of Option Pricing" forthcoming in Handbook of Financial Econometrics by Y. Aït-Sahalia and L. P. Hansen, Eds., North-Holland: Amsterdam.

Garcia, R., R. Luger and E. Renault (2001) "Asymmetric Smiles, Leverage Effects, and Structural Parameters" Working paper, CIRANO.

Hagiwara, M. and M. A. Herce (1997) "Risk Aversion and Stock Price Sensitivity to Dividends" American Economic Review 87, 738-745.

Jackwerth, J. C. (2000) "Recovering Risk Aversion from Option Prices and Realized Returns" Review of Financial Studies 13, 433-451.

Merton, R. C. (1973) "Theory of Rational Option Pricing” The Bell Journal of Economics and Management Science 4, 141-183.

Sukhomlin, N. B. (2007) "Conservation Law of Strike Price and Inversion of the BlackScholes Formula” Russian Physics Journal 50, $741-743$ (Translated from Russian). 


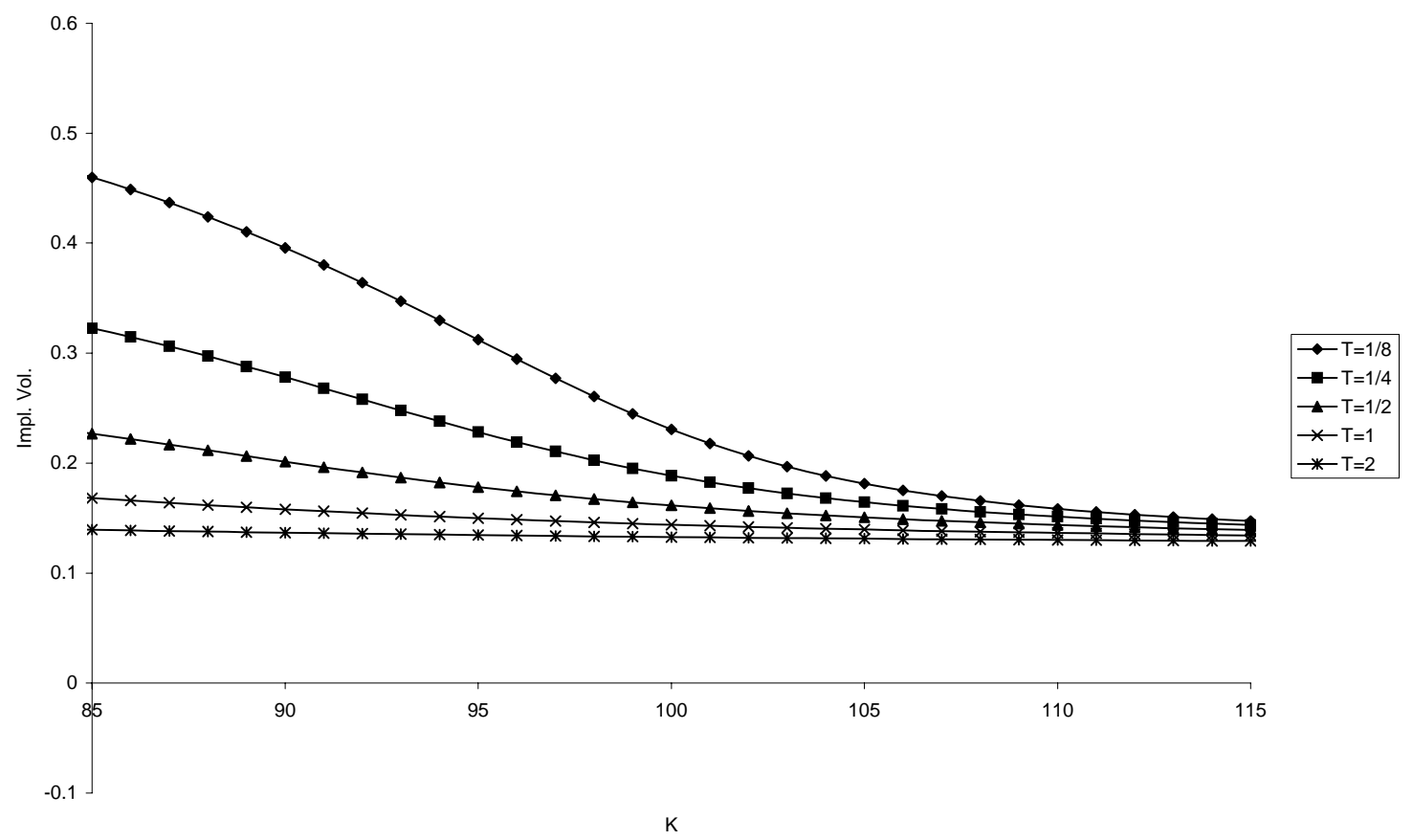

Figure 1: The implied volatility at time 0 as a function of the strike price $(K)$, for various maturities, given that the probability of a low growth rate is $90 \%(v=0.9)$. The values of the other inputs are: $S(0)=100, \beta=0.05, \gamma=3, \theta_{1}=0.02, \theta_{2}=0.03$. The true volatility is constant at $12 \%$.

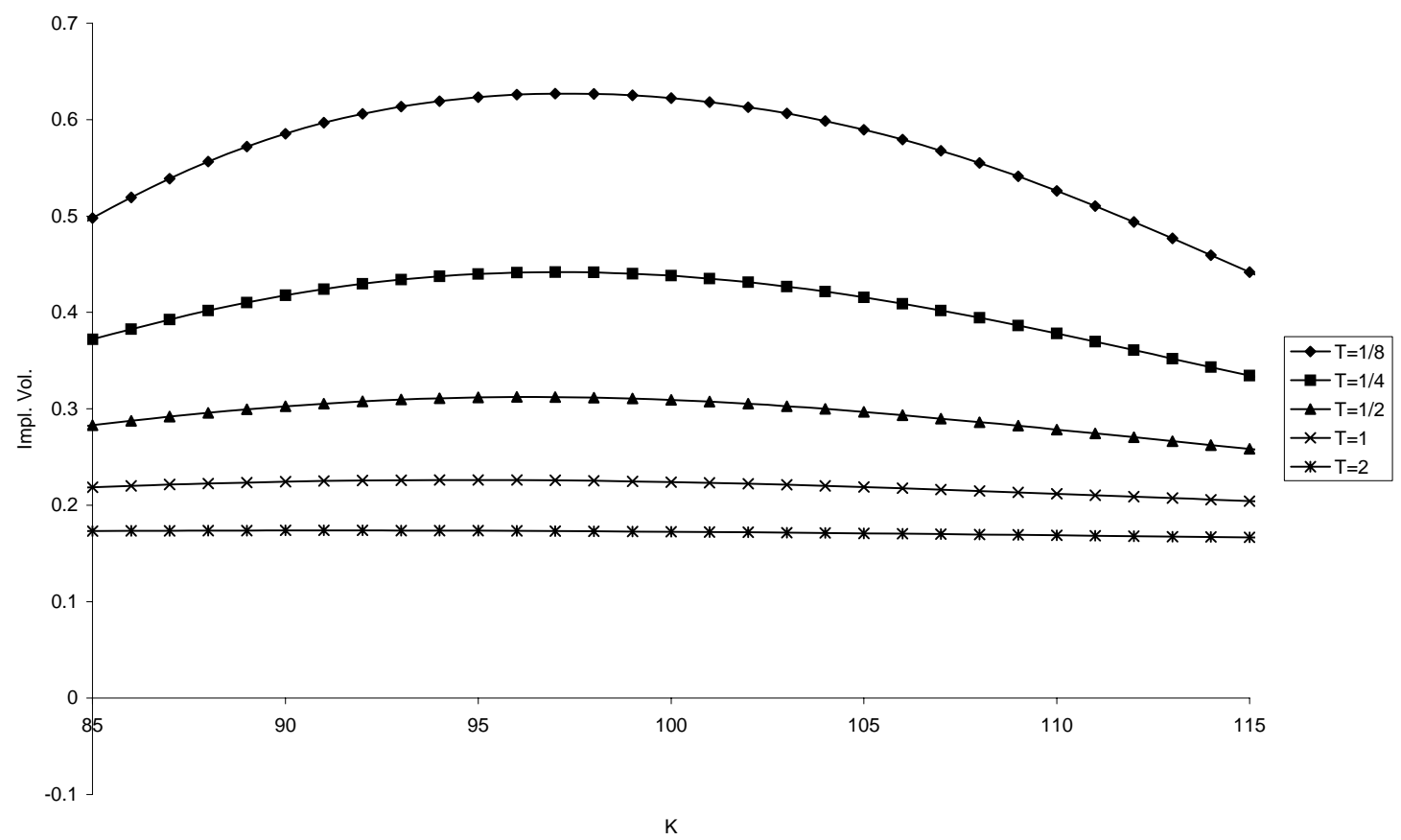

Figure 2: The implied volatility at time 0 as a function of the strike price $(K)$, for various maturities, given that the probability of a low growth rate is $50 \%(v=0.5)$. The values of the other inputs are: $S(0)=100, \beta=0.05, \gamma=3, \theta_{1}=0.02, \theta_{2}=0.03$. The true volatility is constant at $12 \%$. 


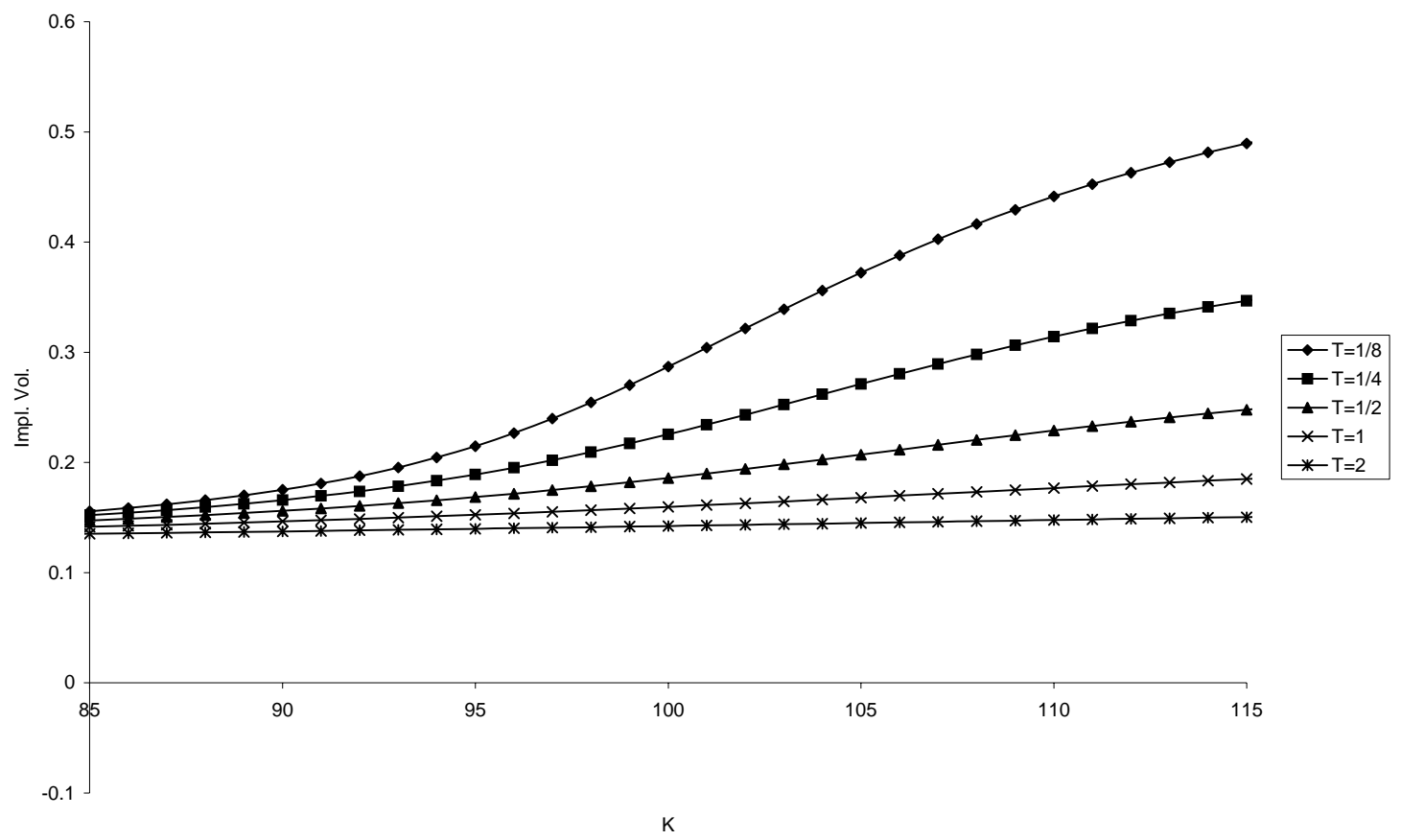

Figure 3: The implied volatility at time 0 as a function of the strike price $(K)$, for various maturities, given that the probability of a low growth rate is $10 \%(v=0.1)$. The values of the other inputs are: $S(0)=100, \beta=0.05, \gamma=3, \theta_{1}=0.02, \theta_{2}=0.03$. The true volatility is constant at $12 \%$.

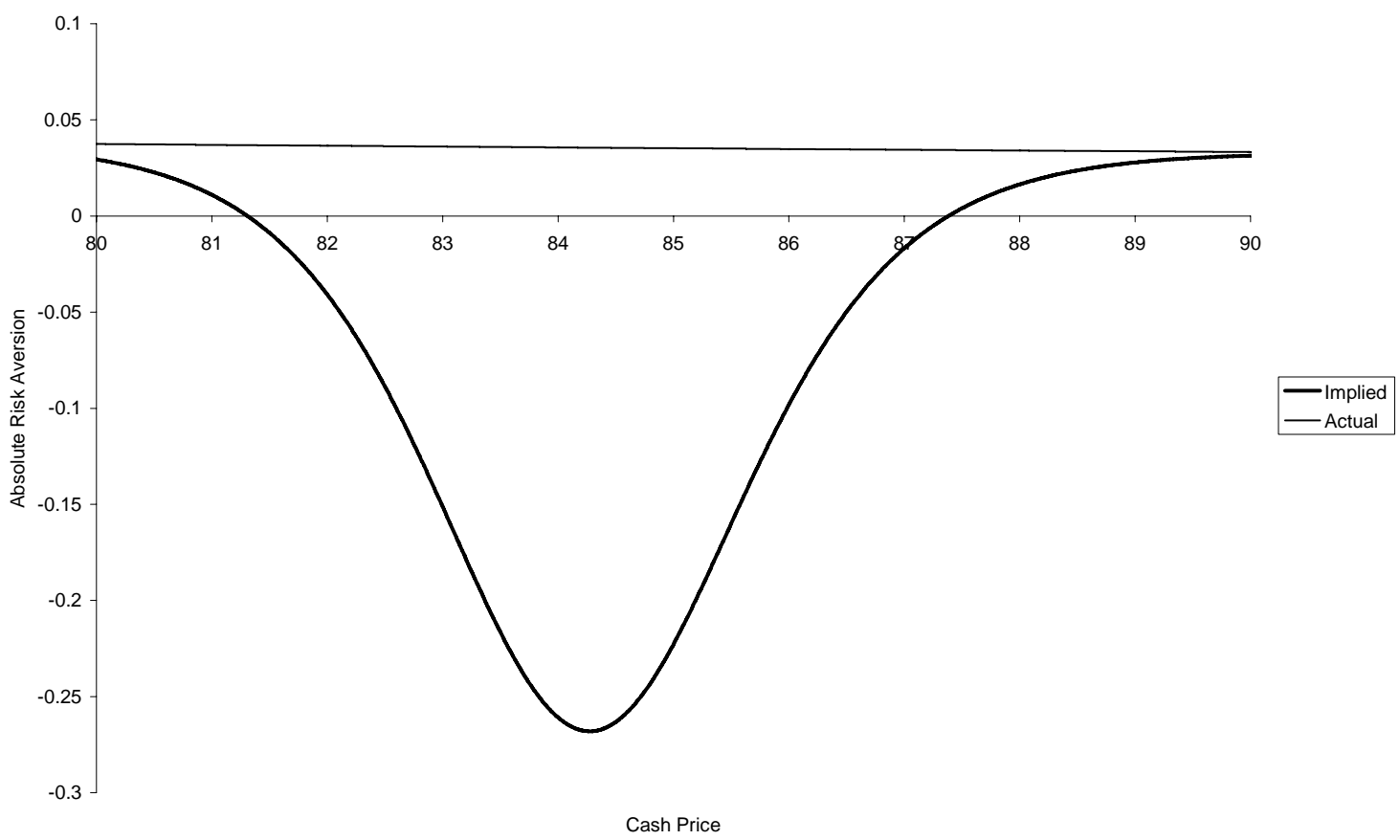

Figure 4: Implied and actual absolute risk aversion as a function of cash price $\left(S_{T}\right)$. The values of the inputs are: $S(0)=100, \beta=0.05, \gamma=3, \theta_{1}=0.02, \theta_{2}=0.03, v=0.9, T=1 / 4, \sigma_{D}=0.12$. 


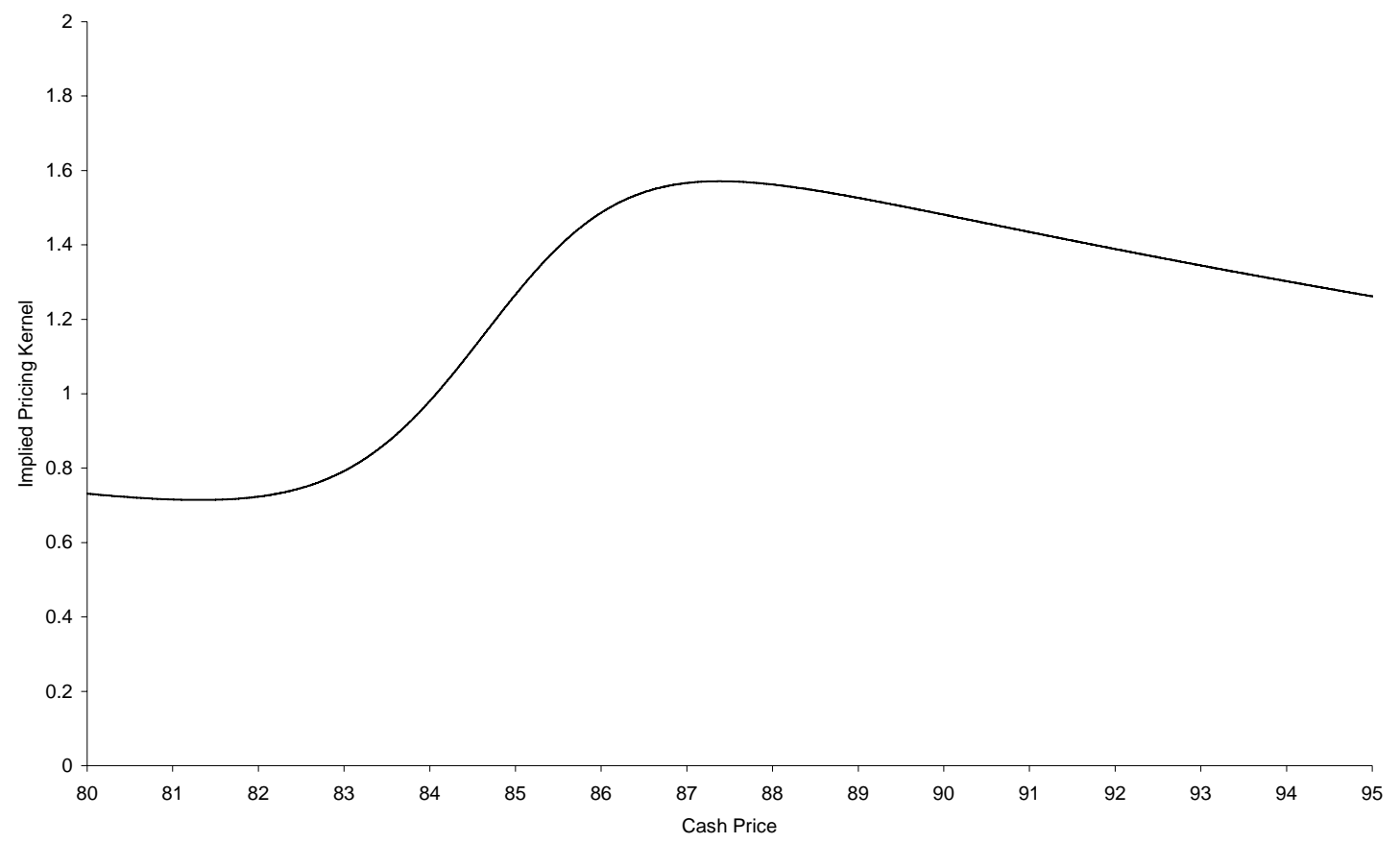

Figure 5: The implied pricing kernel as a function of cash price $\left(S_{T}\right)$. The values of the inputs are: $S(0)=100, \beta=0.05, \gamma=3, \theta_{1}=0.02, \theta_{2}=0.03, \nu=0.9, T=1 / 4, \sigma_{D}=0.12$.

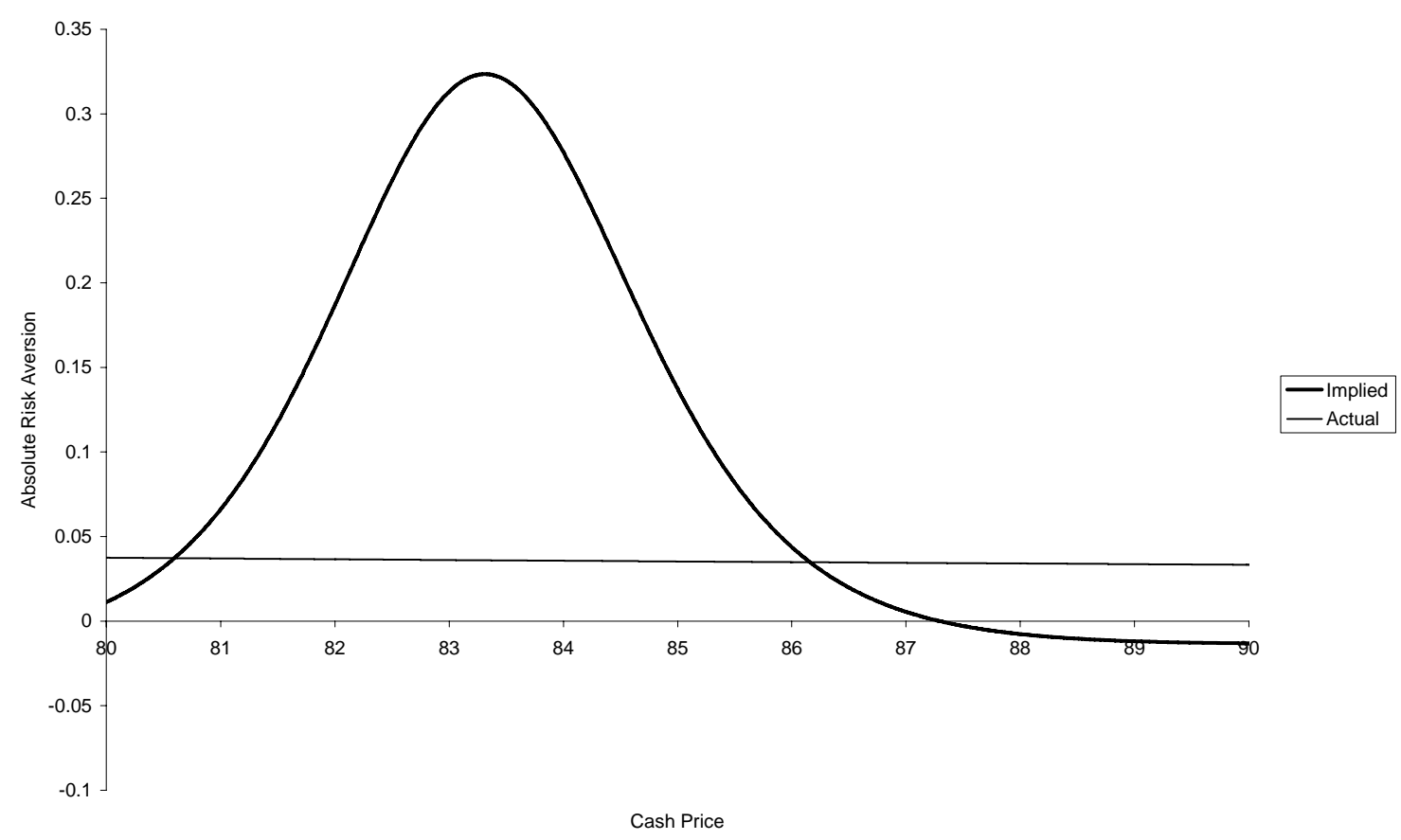

Figure 6: Implied and actual absolute risk aversion as a function of cash price $\left(S_{T}\right)$, given that the econometrician's estimate is $\hat{v}=0.95$. The values of the inputs are: $S(0)=100, \beta=0.05, \gamma=3$, $\theta_{1}=0.02, \theta_{2}=0.03, v=0.9, T=1 / 4, \sigma_{D}=0.12$. 